\title{
Controlling the Reversible Assembly of Liposomes through a Multistimuli Responsive Anchored DNA
}

\author{
Silvia Hernández-Ainsa, ${ }^{*}{ }^{\dagger}$ Maria Ricci, ${ }^{\dagger}$ Lloyd Hilton, ${ }^{\dagger}$ Anna Aviñó, ${ }^{\ddagger}$ Ramon Eritja, ${ }^{\ddagger}$ \\ and Ulrich F. Keyser \\ ${ }^{\dagger}$ Cavendish Laboratory, Department of Physics, University of Cambridge, JJ Thomson Avenue, Cambridge, CB3 0HE, United \\ Kingdom \\ ${ }^{*}$ IQAC-CSIC, CIBER-BBN Networking Centre on Bioengineering, Biomaterials and Nanomedicine, c/Jordi Girona 18-26, 08034 \\ Barcelona, Spain
}

\section{Supporting Information}

ABSTRACT: We present a novel approach to reversibly control the assembly of liposomes through an anchored multistimuli responsive DNA oligonucleotide decorated with an azobenzene moiety $\left(\mathrm{AZO}-\mathrm{ON}_{1}\right)$. We show that liposomes assembly can be simultaneously controlled by three external stimuli: light, $\mathrm{Mg}^{2+}$, and temperature. (i) Light alters the interaction of $\mathrm{AZO}-\mathrm{ON}_{1}$ with liposomes, which influences DNA coating and consequently liposomes assembly. (ii) $\mathrm{Mg}^{2+}$ induces the assembly, hence variation in its concentration enables for reversibility. (iii) Double-stranded $\mathrm{AZO}-\mathrm{ON}_{1}$ is more efficient than singlestranded $\mathrm{AZO}-\mathrm{ON}_{1}$ in triggering the assembly of liposomes and temperature has been used for controllable assembly through DNA thermal denaturation. Our multiresponsive AZO-ON represents a unique example in which multiple stimuli can be simultaneously applied to regulate the reversible assembly of liposomes.

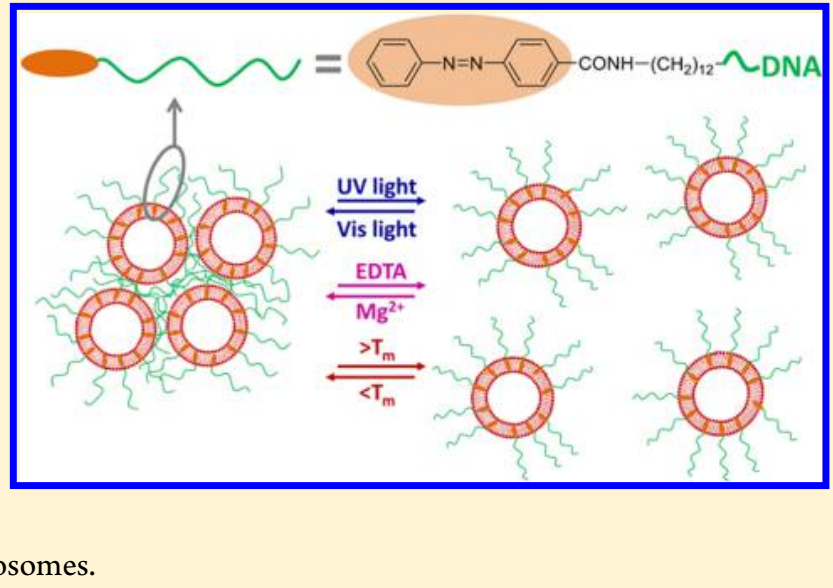

KEYWORDS: DNA, liposomes, self-assembly, azobenzene, stimuli-responsive materials

$\mathrm{A}^{\mathrm{n}}$ $\mathrm{n}$ attractive characteristic of self-assembled molecular materials is the possibility of tuning their arrangement upon activation by external stimuli. ${ }^{1,2}$ DNA has emerged as a versatile building block for the construction of well-defined nanostructures $^{3,4}$ as well as diverse dynamic supramolecular systems such as hydrogels ${ }^{5-7}$ or nanoparticle arrangements ${ }^{8-10}$ whose assembly can be externally regulated in a reversible fashion. Many of these DNA-based tunable supramolecular nanostructures are based on altering base pairs bonding, thus temperature is used as an effective stimulus to reversibly control their assembly through thermal DNA denaturation. ${ }^{11-13}$ Cations and $\mathrm{pH}$ have been also widely used to control DNAassemblies through the reversible formation of G-quadruplexes or i-motifs. ${ }^{14,15}$ The attachment of light responsive azobenzene moieties into the DNA backbone has resulted in an effective strategy to influence base-pairing interactions and hence to guide supramolecular arrangement through light. ${ }^{16,17}$ In most of these systems, the reversible assembly is achieved using a single independent stimulus. Designing dynamic assemblies simultaneously sensitive to several stimuli is challenging but desirable as this multi-triggered response can introduce new opportunities for smart nanostructures fabrication. ${ }^{18,19}$ In this Letter, we present a new approach to create multiresponsive and reversible assembled molecular systems based on large unilamellar lipid vesicles (LUVs) whose assembly is controlled by an anchored oligonucleotide (ON) that simultaneously responds to light, $\mathrm{Mg}^{2+}$ concentration, and temperature. LUVs assembly by anchored DNA has been traditionally achieved following two main strategies. One involves the modification of DNA strands with hydrophobic moieties at both ends so that initially they are doubly anchored to the same liposome but once hybridized with the complementary strands they become stiffer and hence are able to tether different LUVs. ${ }^{9,20-22}$ The other strategy consists of anchoring to liposomes DNA strands modified with a single hydrophobic moiety in one end and then mixing them with other liposomes bearing complementary strands, which results in LUVs assembly. ${ }^{13,23,24}$ Our novel approach involves the anchoring of a single type of ON that produces LUVs assembly due to the hybridization of a selfcomplementary fragment. Importantly our triple stimulitriggered and reversible strategy is unprecedented to achieve control over the assembly of liposomes.

Assembly studies were done with LUVs of $200 \mathrm{~nm}$ in diameter prepared with 2-oleoyl-1-palmitoyl-sn-glycero-3-phosphocholine (POPC) lipids ${ }^{25}$ in a solution containing $20 \mathrm{mM}$

Received: April 20, 2016

Revised: June 23, 2016

Published: July 1, 2016 
$\mathrm{MgCl}_{2}$ buffered at $\mathrm{pH}=8$ with $1 \times \mathrm{TE}$. An oligonucleotide $\left(\mathrm{ON}_{1}\right.$, Figure 1a) bearing a cholesterol (Chol) modification at

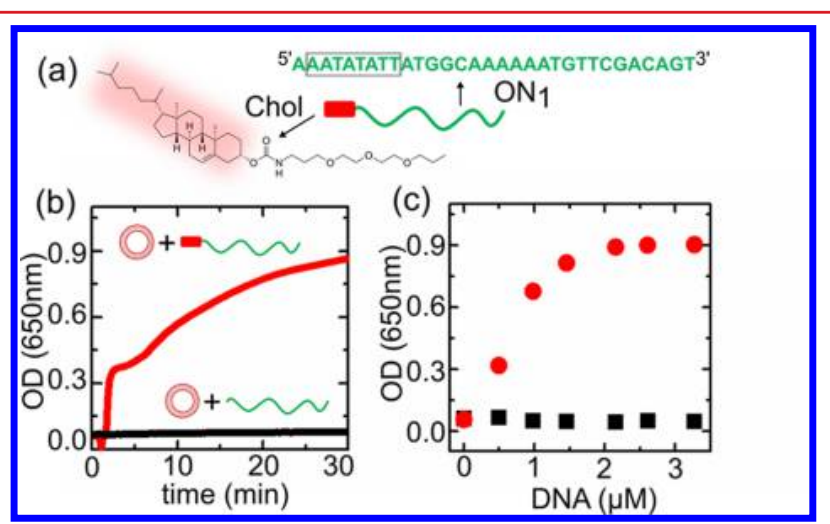

Figure 1. Assembly of LUVs with cholesterol-modified DNA. (a) Schematic representation of Chol- $\mathrm{ON}_{1}$. Chol is attached at the $5^{\prime}$ end. The sequence of $\mathrm{ON}_{1}$ and the chemical structure of $\mathrm{Chol}$ anchor are shown. The self-complementary part of $\mathrm{ON}_{1}$ is marked in gray. (b) Evolution of OD650 with time for LUVs after the addition of $2 \mu \mathrm{M}$ of $\mathrm{Chol}^{-\mathrm{ON}_{1}}$ (red) or $2 \mu \mathrm{M}$ of the same oligonucleotide without cholesterol, NA-ON 1 (black). (c) Evolution of OD650 for LUVs with

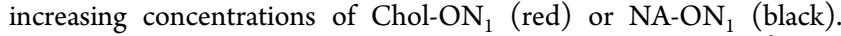
Concentration of LUVs in (b) and (c) was $1 \mathrm{mM}$ in $20 \mathrm{mM} \mathrm{Mg}^{2+}$ (1X TE).

the 5 '-end $\left(\mathrm{Chol}-\mathrm{ON}_{1}\right)$ was added to the solution of POPC LUVs $(1 \mathrm{mM})$ to a final concentration of $2 \mu \mathrm{M}$. Chol is a hydrophobic residue that has been previously used to anchor ONs to liposomes. ${ }^{26-29}$ Upon addition of Chol-ON , $^{\text {, there was }}$ a significant increase in the turbidity of the suspension caused by LUVs aggregation. This process was followed in time by measuring the evolution of the absorbance at $650 \mathrm{~nm}$ (optical density, OD650) $)^{30}$ after addition of $2 \mu \mathrm{M}$ of Chol-ON (red $_{1}$ curve in Figure 1b). On the contrary, no change in OD650 was observed if the LUVs were incubated with $\mathrm{ON}_{1}$ lacking of cholesterol anchor $\left(\mathrm{NA}-\mathrm{ON}_{1}\right.$, black curve in Figure $\left.1 \mathrm{~b}\right)$. The LUVs assembly is thus not produced by nonspecific adsorption of single-stranded (ss) DNA onto LUVs surface mediated by $\mathrm{Mg}^{2+} \cdot{ }^{2}$ No turbidity was observed either when LUVs were incubated with a Chol-ON bearing 31 thymines where no selfcomplementary base pairing can be produced (Figure S6). Therefore, we unambiguously assign the LUVs assembly to the base pair interactions produced by the self-complementary domain present in $\mathrm{ON}_{1}$ (Figure 1a). ${ }^{32}$ An increase in OD650 (Figure 1c, red data) and in particle size (Figure S8) was observed when LUVs were incubated for $5 \mathrm{~min}$ with increasing concentrations of Chol- $\mathrm{ON}_{1}$. This indicates that the assembly process is more efficient for larger $\mathrm{Chol}-\mathrm{ON}_{1} / \mathrm{LUV}$ s relative ratios due to the increasing number of $\mathrm{ONs}$ bound onto the vesicles surface. This increase was also observed when the Chol modification was inserted at the 3 '-end of the $\mathrm{ON}_{1}$ (Figure S7), which indicates that positioning the self-complementary fragment at either side of the strand enables self-hybridization and thus is effective to produce LUVs assembly. On the contrary, no change in OD650 is detected in the case of LUVs incubated with increasing concentrations of NA-ON (Figure $_{1}$ 1c, black data).

We thus engineered an $\mathrm{ON}$ bearing an anchor whose hydrophobicity could be tuned by light in order to influence its ability to insert into the liposomes and hence to control the aggregation process. Specifically, we synthesized an ON bearing a $5^{\prime}-\mathrm{C}_{12}$ aliphatic chain with an azobenzene $(\mathrm{AZO})$ residue at the end $\left(\mathrm{AZO}-\mathrm{ON}_{1}\right)$ (Figure $\left.2 \mathrm{a}\right)$. We proved that $\mathrm{AZO}-\mathrm{ON}_{1}$ is able to interact with lipid vesicles using fluorescent confocal microscopy by incubating POPC giant unillamelar vesicles (GUVs) with Cy3-labeled double stranded (ds) AZO-ON (Figure S10). ${ }^{33} \mathrm{AZO}$ undergoes reversible isomerization from a trans to a cis conformation upon irradiation with UV light and

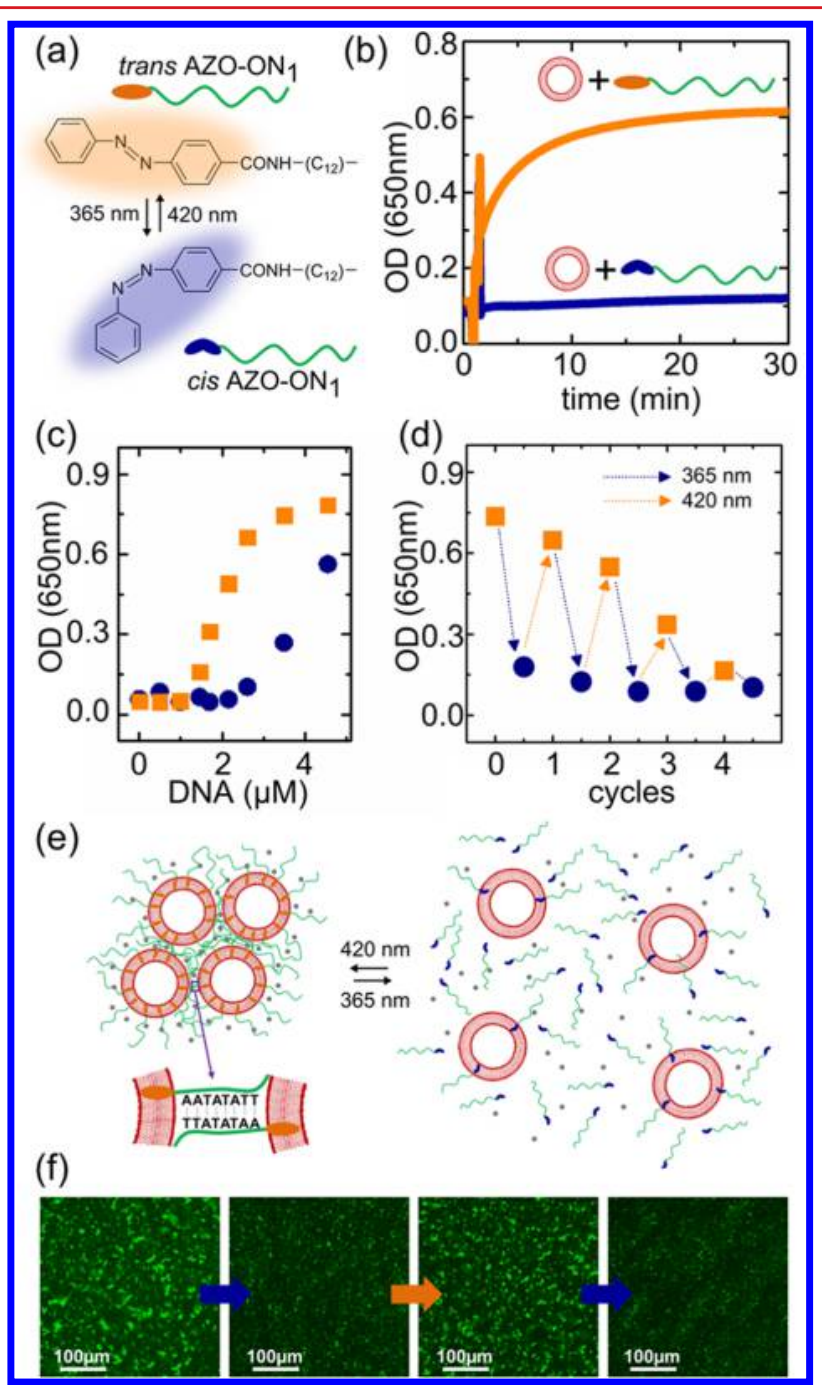

Figure 2. Light reversible LUVs assembly using AZO-ON ${ }_{1}$. (a) Schematic representation of the AZO-ON $\mathrm{N}_{1}$ including the chemical structure of $\mathrm{AZO}$ anchor in its trans (orange) and cis (blue) isomers. (b) Evolution of OD650 with time for LUVs after the addition of trans $\mathrm{AZO}-\mathrm{ON}_{1}$ (orange) or cis AZO-ON ${ }_{1}$ (blue). (c) Evolution of OD650 for LUVs with increasing concentrations of trans $\mathrm{AZO}-\mathrm{ON}_{1}$ (orange)

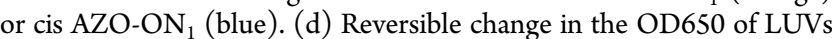
incubated with $\mathrm{AZO}-\mathrm{ON}_{1}$ triggered by light irradiation. Orange data are the OD650 values displayed by the LUVs in the presence of trans AZO-ON ${ }_{1}$ (after irradiation at $420 \mathrm{~nm}$, orange arrows) whereas blue data show the OD650 values shown by the LUVs in the presence of cis AZO-ON ${ }_{1}$ (after irradiation at $365 \mathrm{~nm}$, blue arrows). (e) Schematic representation of the reversible LUVs assembly process upon light irradiation. LUVs are shown as red circumferences. AZO-ON $\mathrm{ON}_{1}$ is represented as a green line ending with an elongated circle either orange (trans) or blue (cis). Gray circles represent $\mathrm{Mg}^{2+}$. (f) Images taken by confocal fluorescence microscopy showing the reversible evolution in size of the LUVs aggregates upon light irradiation. Blue arrows represent irradiation at $365 \mathrm{~nm}$ and the orange arrow indicates irradiation at $420 \mathrm{~nm}$. 
from cis to trans upon irradiation with visible light (Figure 2a and Figure S9). ${ }^{34}$ This reversible change in conformation has been previously used to control the hybridization of DNA through light irradiation. ${ }^{16,17}$ Importantly, this change in conformation is accompanied by a modification in the polarity of the AZO and this variation has been reported to influence its hydrophobicity. ${ }^{35-37}$ The addition of trans AZO-ON ${ }_{1}(2 \mu \mathrm{M})$ to a solution of POPC-LUVs $(1 \mathrm{mM})$ gave rise to an increase in the OD650 (Figure 2b, orange curve) unlike the addition of cis $\mathrm{AZO}-\mathrm{ON}_{1}$ (Figure 2b, blue curve). As shown in Figure 1c, the LUVs assembly is enhanced by increasing the concentration of Chol-ON $\mathrm{N}_{1}$. We observed a similar trend in the case of trans and cis AZO-ON $\mathrm{ON}_{1}$ (Figure 2c, orange and blue data, respectively). However, the maximum OD650 value is reached at different DNA concentrations for Chol-ON $\mathrm{CN}_{1}$, trans-AZO $\mathrm{ON}_{1}$ and cis-AZO $\mathrm{ON}_{1}$. This indicates that the efficacy of the anchors for LUVs attachment varies in the order Chol $>$ trans$\mathrm{AZO}>$ cis-AZO. We also observed that the LUVs assembly is more efficient when increasing the binding strength of the selfcomplementary fragment present in the AZO-ON (Figures S11 and S12). Therefore, both the efficiency of the hydrophobic anchor and the strength of the self-complementary fragment influence the ability to produce LUVs assembly.

Then we investigated the effect that the isomerization of the AZO anchor had in the LUVs assembly. As shown in Figure 2d, a decrease in the OD650 of the solution is observed when irradiating with light at $365 \mathrm{~nm}$ the trans AZO-ON $\mathrm{N}_{1}$ LUVs assembled system, which indicates a light-triggered LUVs disassembly process (blue data). Interestingly, reassembly can be produced by irradiating at $420 \mathrm{~nm}$, as the increase in OD650 denotes (Figure $2 \mathrm{~d}$, orange data).

This assembly disassembly process (Figure $2 \mathrm{e}$ ) is reversible up to four cycles (Figure 2d). The reversible aggregation was also confirmed with confocal microscopy by incubating the LUVs with AZO-ON 1 labeled with cyto 9 (see Figure $2 \mathrm{f}$ and Figure S13). Large lipid aggregates were observed in the presence of the trans $\mathrm{AZO}-\mathrm{ON}_{1}$ isomer (panels 1 and 3 in Figure 2f) that became smaller upon irradiation with $365 \mathrm{~nm}$ wavelength light (panels 2 and panel 4 in Figure 2f).

Next we investigated the possibility to control the LUVs assembly by modifying the concentration of $\mathrm{Mg}^{2+}$. LUVs (1 $\mathrm{mM})$ were incubated with trans AZO-ON $1(2 \mu \mathrm{M})$ at different concentrations of $\mathrm{Mg}^{2+}$ from 0 to $40 \mathrm{mM}$ (Figure 3a). OD650 values increased as the concentration of $\mathrm{Mg}^{2+}$ was raised. We assign this effect to the stabilization of the complementary basepair interactions provided by $\mathrm{Mg}^{2+}$ which favors the selfcomplementary recognition of the strands coating the liposomes $^{38}$ as well as a decrease in the Debye length which enhances LUVs assembly. This $\mathrm{Mg}^{2+}$ dependence allowed for reversibly controlling the LUVs assembly by EDTA. The reversible changes are evidenced by the OD650 data displayed in Figure $3 \mathrm{~b}$ and the process is schematically presented in Figure 3c.

Finally we show that even ds trans AZO-ON ${ }_{1}$ (ss trans AZO$\mathrm{ON}_{1}$ hybridized with its complementary strand) is able to induce LUVs assembly. Addition of ds trans AZO-ON ${ }_{1}(2 \mu \mathrm{M})$ to LUVs $(1 \mathrm{mM})$ produced an increase in turbidity (Figure S15a). In this case, the assembly is clearly not mediated by the complementary interaction of the bases but by some other phenomena. Blunt-end attachment has been previously described to be the most likely origin of the aggregation of nanoparticles coated with ds DNA. ${ }^{39}$ However, after recording the OD650 evolution with time in LUVs coated with ds trans

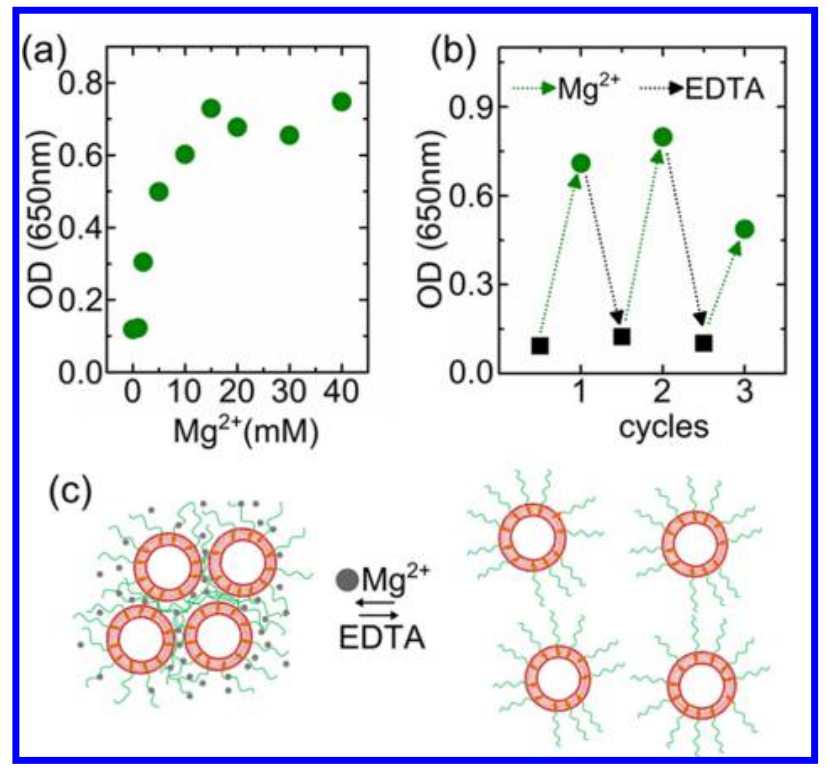

Figure 3. $\mathrm{Mg}^{2+}$ driven LUVs assembly. (a) Evolution of OD650 with the concentration of $\mathrm{Mg}^{2+}$ present in solution for LUVs incubated with trans AZO-ON ${ }_{1}$. (b) Reversible change in the OD650 nm for LUVs incubated with trans AZO-ON $\mathrm{N}_{1}$ in the presence of $\sim 25 \mathrm{mM} \mathrm{Mg}{ }^{2+}$ (green data) or absence of $\mathrm{Mg}^{2+}$ (black data). Green and black arrows represent the addition of $\mathrm{Mg}^{2+}$ and EDTA respectively. (c) Schematic representation of the reversible LUVs assembly process upon variation of $\mathrm{Mg}^{2+}$ concentration. LUVs are shown as red circumferences. ss trans AZO-ON ${ }_{1}$ is represented as a green line. Gray circles represent $\mathrm{Mg}^{2+}$.

AZO-ON $\mathrm{ON}_{1}$ with a single-base mismatch at the distal end, assembly is also observed (Figure $\mathrm{S} 15 \mathrm{~b}-\mathrm{d}$ ). This indicates that the aggregation mechanism in our system is not uniquely caused by end-to end DNA stacking. We suggest that the assembly is produced by nonspecific interactions of the dsDNA coating the LUVs helped by the presence of $\mathrm{Mg}^{2+}$. Similar aggregation processes have been described in particles composed by poly $(N$-isopropylacrylamide $)$ grafted with DNA. ${ }^{40,41}$

In order to clarify the role of $\mathrm{ON}$-coating density, we investigated the variation in OD650 and hence the ability to produce LUVs assembly produced by different concentrations

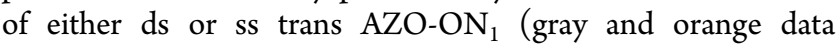
respectively, Figure 4a). It is observed that the concentration of ss trans $\mathrm{AZO}-\mathrm{ON}_{1}$ required to produce the assembly is higher than in the case of ds trans AZO-ON ${ }_{1}$. Contrary, ds NA-ON showed no LUVs assembly in this range of concentrations (Figure S14), which evidence the need of a hydrophobic anchor for enabling the assembly process excluding a mechanism purely controlled by electrostatics.

The assembly ability produced by ds trans AZO-ON allowed for the introduction of a temperature-controlled reversible LUVs assembly strategy. When measuring the absorbance at $260 \mathrm{~nm}$ (abs260) while increasing temperature in a mixture containing LUVs $(1 \mathrm{mM})$ and ds trans AZO-ON $(1 \mu \mathrm{M})$, a sharp drop is detected (Figure $4 \mathrm{~b}$, dark black curve). This drop occurs at the melting temperature $\left(T_{\mathrm{m}}\right)$ of ds trans $\mathrm{AZO}-O N_{1}$ (Figure 4c, dark blue curve) and it is neither


red curve) nor in the case of bare LUVs (Figure S16). This drop in abs 260 corresponds to a decrease in the turbidity of the solution that is produced by the disassembly of the LUVs ${ }^{9,13}$

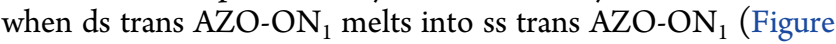




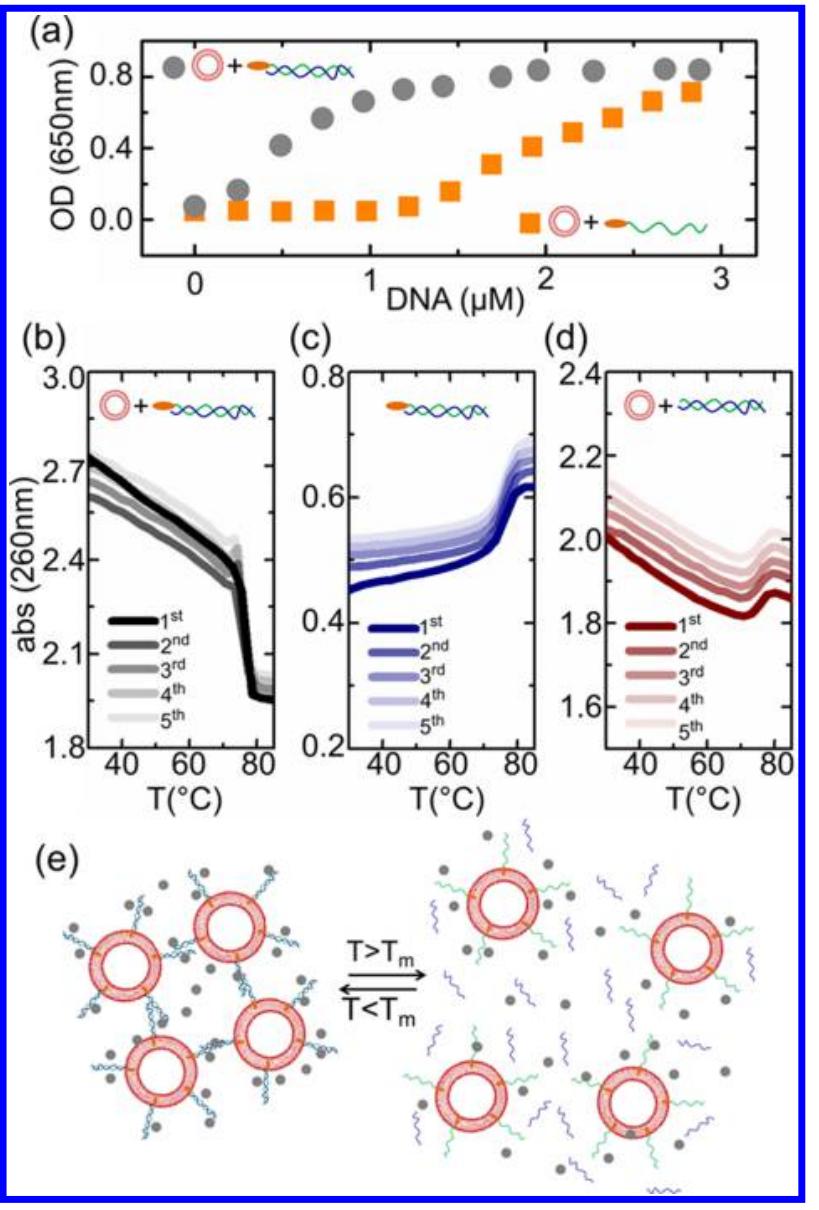

Figure 4. Temperature-controlled LUVs assembly. (a) Evolution of OD650 for LUVs after the incubation with increasing concentrations of either ss trans $\mathrm{AZO}-\mathrm{ON}_{1}$ (orange) or ds trans AZO-ON $\mathrm{ON}_{1}$ (gray). (b) Evolution of the abs $260 \mathrm{~nm}$ with T of LUVs incubated with ds

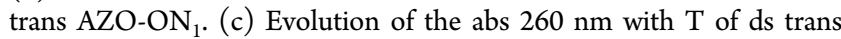
AZO-ON ${ }_{1}$ in the absence of LUVs. (d) Evolution of the abs $260 \mathrm{~nm}$ with $\mathrm{T}$ of LUVs incubated with ds NA-ON ${ }_{1}$. Note than in $(b, d)$ abs $260 \mathrm{~nm}$ refers to apparent absorbance because the lack of light transmitted is the result of the scattered light produced by the presence of particles as well as of the absorption produced by the presence of DNA. Note that in $(b-d)$ five subsequent heating cycles are shown in each graph. (e) Schematic representation of the reversible LUVs assembly process triggered by temperature. LUVs are shown as red circumferences. ss trans $\mathrm{AZO}-\mathrm{ON}_{1}$ is represented as a green line and its complementary strand as a blue line. Gray circles represent $\mathrm{Mg}^{2+}$.

4e). Interestingly, this process is reversible as shown in Figure $4 \mathrm{~b}$ where up to five subsequent cycles were recorded. The reversible assembly is only produced at concentrations of ds trans AZO-ON $\mathrm{ON}_{1}$ between 0.5 and $1.5 \mu \mathrm{M}$ (Figure S18), range in which the OD650 values for ss or ds trans $\mathrm{AZO}-\mathrm{ON}_{1}$ are different (Figure 4a). This thermal-driven disassembly process was studied in three additional ds AZO-ONs of the same length as $\mathrm{AZO}-\mathrm{ON}_{1}$ (31bp) (Table S5) as well as in other $\mathrm{ds}$ derivatives resulting from the partial hybridization of ss trans AZO-ON ${ }_{1}$ with complementary strands being 22,14 , and 11 nucleotides (NTS) long (Table S6). In all cases, we observed that the temperature of LUVs disassembly matches with the Tm of the employed sequences (Tables S5 and S6 and Figure $\mathrm{S} 19)$. We also demonstrated that $\mathrm{AZO}-\mathrm{ON}_{1}$ was optimal for the detection of a single base mismatch in complementary ONs of 14 or 13 NTS in length (Tables S7 and S8 and Figures S20 and S21). Our system thus allows for the detection of a range of oligonucleotide targets with different lengths as well as single base mismatches in short ONs by employing a unique DNA probe with a single hydrophobic anchor $\left(\mathrm{AZO}-\mathrm{ON}_{1}\right)$. This sensing ability is highly relevant since it may enable to identify point mutations in genes for the diagnosis of inherited genetic diseases such as cystic fibrosis, sickle cell anemia, or thalassemia as well as to detect single nucleotide polymorphisms (SNP) ${ }^{42}$ in an easy optically measured way. Our approach represents a simplified strategy and a versatile method respect to other previously reported oligonucleotide sensing systems based on the assembly of DNA-modified liposomes in which either the DNA-probe has to carry two hydrophobic anchors ${ }^{9}$ or two DNA probes with single anchors tethering two liposomes are required. ${ }^{13}$

In conclusion, we have presented a novel dynamic supramolecular system based on liposomes whose assembly can be controlled simultaneously by the action of three different stimuli (light, $\mathrm{Mg}^{2+}$, and temperature) due to a multiresponsive anchored DNA oligonucleotide. Our approach is unprecedented for the achievement of multicontrollable and reversible assembly of liposomes. Our system presents novel capabilities as sensor for the detection of nucleic acids and it opens up new possibilities for the creation of stimuli-responsive nanocontainers $^{7,43,44}$ in which different analytes such as DNA, RNA, or proteins could get trapped and released into the DNA-liposome network on demand by the action of several stimuli.

\section{ASSOCIATED CONTENT}

\section{S Supporting Information}

The Supporting Information is available free of charge on the ACS Publications website at DOI: 10.1021/acs.nanolett.6b01618.

Experimental details, Figures S1-S21, and Tables S1S8. (PDF)

\section{AUTHOR INFORMATION}

\section{Corresponding Author}

*E-mail: smh80@cam.ac.uk.

\section{Author Contributions}

The manuscript was written through contributions of all authors. All authors have given approval to the final version of the manuscript.

\section{Notes}

The authors declare no competing financial interest.

\section{ACKNOWLEDGMENTS}

S.H.A. acknowledges support from a Herchel Smith postdoctoral fellowship. M.R. acknowledges support from an Early Postdoc Mobility fellowship of Swiss National Science Foundation. A.A. and R.E. acknowledge support from MINECO, Spain, CTQ2014-52588-R. U.F.K. acknowledges support from an ERC Starting Grant Passmembrane 261101.

\section{REFERENCES}

(1) Stuart, M. A. C.; Huck, W. T.; Genzer, J.; Müller, M.; Ober, C.; Stamm, M.; Sukhorukov, G. B.; Szleifer, I.; Tsukruk, V. V.; Urban, M. Nat. Mater. 2010, 9, 101

(2) Rybtchinski, B. ACS Nano 2011, 5, 6791. 
(3) Zhang, F.; Nangreave, J.; Liu, Y.; Yan, H. I.Am. Chem. Soc. 2014, 136, 11198.

(4) Aldaye, F. A.; Palmer, A. L.; Sleiman, H. F. Science 2008, 321, 1795.

(5) Li, J.; Zheng, C.; Cansiz, S.; Wu, C.; Xu, J.; Cui, C.; Liu, Y.; Hou, W.; Wang, Y.; Zhang, L. I. Am. Chem. Soc. 2015, 137, 1412.

(6) Lu, C.-H.; Guo, W.; Hu, Y.; Qi, X.-J.; Willner, I. I. Am. Chem. Soc. 2015, 137, 15723.

(7) Xiong, X.; Wu, C.; Zhou, C.; Zhu, G.; Chen, Z.; Tan, W. Macromol. Rapid Commun. 2013, 34, 1271.

(8) Mirkin, C. A.; Letsinger, R. L.; Mucic, R. C.; Storhoff, J. I. Nature 1996, 382, 607.

(9) Jakobsen, U.; Simonsen, A. C.; Vogel, S. I. Am. Chem. Soc. 2008, $130,10462$.

(10) Rogers, W. B.; Shih, W. M.; Manoharan, V. N. Nat. Rev. Mater. 2016, 1, 16008.

(11) Jin, R.; Wu, G.; Li, Z.; Mirkin, C. A.; Schatz, G. C. I. Am. Chem. Soc. 2003, 125, 1643.

(12) Li, Z.; Zhu, Z.; Liu, W.; Zhou, Y.; Han, B.; Gao, Y.; Tang, Z. I. Am. Chem. Soc. 2012, 134, 3322.

(13) Dave, N.; Liu, J. ACS Nano 2011, 5, 1304.

(14) Liu, D.; Bruckbauer, A.; Abell, C.; Balasubramanian, S.; Kang,

D.-J.; Klenerman, D.; Zhou, D. I. Am. Chem. Soc. 2006, 128, 2067.

(15) Sharma, J.; Chhabra, R.; Yan, H.; Liu, Y. Chem. Commun. 2007, 477.

(16) Kuzyk, A.; Yang, Y.; Duan, X.; Stoll, S.; Govorov, A. O.; Sugiyama, H.; Endo, M.; Liu, N. Nat. Commun. 2016, 7, 10591.

(17) Yan, Y.; Chen, J. I.; Ginger, D. S. Nano Lett. 2012, 12, 2530.

(18) Zhuang, J.; Gordon, M. R.; Ventura, J.; Li, L.; Thayumanavan, S. Chem. Soc. Rev. 2013, 42, 7421.

(19) Theato, P.; Sumerlin, B. S.; O’Reilly, R. K.; Epps, T. H., III Chem. Soc. Rev. 2013, 42, 7055.

(20) Zhang, G.; Farooqui, F.; Kinstler, O.; Letsinger, R. L. Tetrahedron Lett. 1996, 37, 6243.

(21) Jakobsen, U.; Vogel, S. Bioconiugate Chem. 2013, 24, 1485.

(22) Jakobsen, U.; Vogel, S. Methods Enzvmol. 2009, 464, 233.

(23) Maruyama, T.; Yamamura, H.; Hiraki, M.; Kemori, Y.; Takata, H.; Goto, M. Colloids Surf., B 2008, 66, 119.

(24) Parolini, L.; Kotar, J.; Di Michele, L.; Mognetti, B. M. ACS Nano 2016, 10, 2392.

(25) Hernández-Ainsa, S.; Muus, C.; Bell, N. A.; Steinbock, L. J.; Thacker, V. V.; Keyser, U. F. Analust 2013, 138, 104.

(26) Langecker, M.; Arnaut, V.; List, J.; Simmel, F. C. Acc. Chem. Res. 2014, 47, 1807.

(27) Banchelli, M.; Betti, F.; Berti, D.; Caminati, G.; Bombelli, F. B.; Brown, T.; Wilhelmsson, L. M.; Nordén, B.; Baglioni, P. I. Phvs. Chem. B 2008, 112, 10942.

(28) Parolini, L.; Mognetti, B. M.; Kotar, J.; Eiser, E.; Cicuta, P.; Di Michele, L. Nat. Commun. 2015, 6, 5948.

(29) Beales, P. A.; Vanderlick, T. K. I. Phvs. Chem. A 2007, 111, 12372.

(30) Hernández-Ainsa, S.; Barberá, J.; Marcos, M.; Serrano, J. L. Soft Matter 2011, 7, 2560.

(31) Gromelski, S.; Brezesinski, G. Langmuir 2006, 22, 6293.

(32) Hurst, S. J.; Hill, H. D.; Mirkin, C. A. I. Am. Chem. Soc. 2008, 130, 12192.

(33) Göpfrich, K.; Zettl, T.; Meijering, A. E.; Hernández-Ainsa, S.; Kocabey, S.; Liedl, T.; Keyser, U. F. Nano Lett. 2015, 15, 3134.

(34) Bandara, H. D.; Burdette, S. C. Chem. Soc. Rev. 2012, 41, 1809.

(35) Sebai, S. C.; Milioni, D.; Walrant, A.; Alves, I. D.; Sagan, S.;

Huin, C.; Auvray, L.; Massotte, D.; Cribier, S.; Tribet, C. Angew. Chem., Int. Ed. 2012, 51, 2132.

(36) Huang, Y.; Kang, H.; Li, G.; Wang, C.; Huang, Y.; Liu, R. $\underline{R S C}$ Adv. 2013, 3, 15909.

(37) Sebai, S. C.; Cribier, S.; Karimi, A.; Massotte, D.; Tribet, $\underline{\text { C. }}$ Langmuir 2010, 26, 14135.

(38) Owczarzy, R.; Moreira, B. G.; You, Y.; Behlke, M. A.; Walder, J.

A. Biochemistry 2008, 47, 5336.
(39) Akiyama, Y.; Shikagawa, H.; Kanayama, N.; Takarada, T.; Maeda, M. Chem. - Eur. I. 2014, 20, 17420.

(40) Ooi, W.-Y.; Fujita, M.; Pan, P.; Tang, H.-Y.; Sudesh, K.; Ito, K.; Kanayama, N.; Takarada, T.; Maeda, M. L. Colloid Interface Sci. 2012, 374, 315.

(41) Fujita, M.; Hiramine, H.; Pan, P.; Hikima, T.; Maeda, $\underline{\text { M. }}$ Langmuir 2016, 32, 1148.

(42) Chang, K.; Deng, S.; Chen, M. Biosens. Bioelectron. 2015, 66, 297.

(43) Rodríguez-Pulido, A.; Kondrachuk, A. I.; Prusty, D. K.; Gao, J.; Loi, M. A.; Herrmann, A. Angew. Chem., Int. Ed. 2013, 52, 1008.

(44) Popescu, M.-T.; Mourtas, S.; Pampalakis, G.; Antimisiaris, S. G.; Tsitsilianis, C. Biomacromolecules 2011, 12, 3023. 